\title{
Decompensated Liver Cirrhosis Infections: Unsuitable Empirical Therapy
}

\author{
Nabila H Ahmed ${ }^{1}$, Marwa Shabana ${ }^{2}$, Soha A Elhawari ${ }^{1}$ \\ ${ }^{1}$ Department of Tropical Medicine, Faculty of Medicine, Zagazig University, Zagazig, \\ Egypt. \\ ${ }^{2}$ Department of Clinical Pathology, Faculty of Medicine, Zagazig University, Zagazig, \\ Egypt
}

Corresponding Author Nabila H Ahmed.

Mobile:

$+201224892292$

E mail:

nabilaahassanahmed. com

Key words:

Infection; antibiotics; resistance; cirrhosis; decompensation .
Background and aim: Infection in patients with decompensated liver cirrhosis is a leading cause of mortality in developing countries such as Egypt; this has paralleled the increase in antibiotics resistance. Knowledge of local antimicrobial susceptibility patterns is critical to decreasing morbidity, hospitalization cost and mortality associated with these infections. Our aim was to evaluate the bacterial resistance profile in ICU admitted patients with decompensated liver cirrhosis.

Methods: This study included 1339 samples from patients with decompensated liver cirrhosis and suspected infection admitted to the intensive care unit - department of Tropical medicine, Zagazig University hospitals, Egypt, between August 2018 and August 2019. Demographical, clinical, microbiological and antimicrobial susceptibility were evaluated.

Results: Out of 1339 total samples collected, 237 positive cultures were

\section{INTRODUCTION}

Antimicrobial resistance (AMR) is microbe`s ability to resist the effects of medication. Antibiotic resistance $(\mathrm{ABR})$ is a sub-type of antimicrobial resistance, referring to development of antibiotic resistance by bacteria. Antibiotics resistant bacteria are difficult to treat, requiring unconventional medications or higher doses of antimicrobials. These additional treatment options are associated with higher incidence of side effects, toxicity and are more costly [1]. Microbes resistant to multiple antimicrobials are termed multidrug resistant (MDR) and those, obtained; the majority $(60.4 \%)$ had urinary tract infection, $18.1 \%$ had sepsis, 9.3\% had chest infection, $8.4 \%$ had spontaneous bacterial peritonitis (SBP), $3.8 \%$ had other infections. One hundred and ninety-nine (199) of the 237 isolates were multi drug resistant (MDR). Among them $72.4 \%$ were gram negative organisms (GNO); Extended Spectrum Beta Lactamase (ESBL) Ecoli and Klebsiella Sp. were $32.2 \%$, and $83.3 \%$ of acinetobacter were MDR. GNO represent $70.9 \%$ and Gram positive organisms (GPO) $29.1 \%$ of isolated bacteria, GPO were $27.6 \%$ of total MDR organisms, mostly Staphlycoccus haemolyticus followed by enterococcus fecalis and fecium. All isolated staphylococcus aureus are methicillin resistant and 25\% of them are MDR.

Conclusion: In patients with decompensated liver cirrhosis and suspected infection, high frequency of multidrug resistance was recorded. Gram negative bacteria showed high resistance to 3rd generation cephalosporins and quinolones.

which considered extensively drug resistant (XDR), pan drug resistant (PDR) or totally drug resistant (TDR) are known as superbugs [2]. The emergence of AMR, which threatens the efficacy of commonly used antibiotic classes is rapidly occurring worldwide, and rendering once easily treatable conditions, potentially fatal [3]. Wrongly prescribed antibiotics contribute to AMR. It has been shown previously that the indication of treatment, agent choice, or antibiotic therapy duration is incorrect in up to half of patients $[4,5]$. 
Bacterial infection is one of the major leading clinical problems in decompensated liver cirrhosis patients, principally in hospitalized patients [6].

Prompt and suitable antibiotic treatment is needed in cirrhosis patients with bacterial infection. Infections may precipitate liver decompensation, and is a documented leading cause of death in these patients. Quinolones and 3 rd generation cephalosporins, once the mainstay of treatment of infections in patients with cirrhosis, are now ineffective in a growing proportion of cases. Antibiotics must be selected according to the type, site and severity of infection, MDR local rate and past antibiotic-use history $[7,8]$.

\section{Aim of Work}

The aim of the work was to evaluate the bacterial resistance profile in ICU admitted patients with decompensated liver cirrhosis.

\section{Patients AND METHODS}

\section{Study design}

Retrospective observational cross sectional study

\section{Definitions}

Multi drugs resistant (MDR) is used to describe organism which is non-susceptible to at least one agent non-susceptible from three or more classes of the antibiotics. Extensive drug resistance (XDR) is used for organisms developed one agent non-susceptibility at least in all but two or fewer antimicrobial classes. Pan drug resistant (PDR) categorized as non-susceptibility to totally available antimicrobial agents. Considering that agents or classes of antimicrobials to which an organism is intrinsically resistant doesn't count in the previous definitions and analysis. Although the names of certain MDROs describe resistance to only one agent (e.g., Methicillin resistant staphylococcus aureus; MRSA, Vancomycin resistant Enterococci; VRE), these pathogens are frequently resistant to most available antimicrobial agents [9].

\section{Patients}

This study was conducted in the Tropical medicine department ICU, Zagazig University Hospitals, between August, 2018 and August, 2019. The study included patients with decompensated liver cirrhosis and suspected infections (signs of infections, any acute changes in a patient's condition) [18]. A total of 237 bacterial isolates out of 1339 samples (blood, respiratory sputum, pleural fluid, urine samples, ascites and abscesses) were included in the study.

\section{Inclusion criteria}

1. Patients with decompensated liver cirrhosis and suspected infection who were admitted in ICU.

2. Biological material samples of great clinical importance (blood samples, urine samples, respiratory secretions (sputum), ascetic fluid, other intra-abdominal fluid collections, skin and wound swabs) [17].

\section{Exclusion criteria}

1. Liver cirrhosis patients without suspected infection

2. Incomplete medical records

3. History of antibiotics taking in the previous 2 weeks pre-admission

4. Biological material samples of lower clinical importance (stool samples, genital secretions, skin, wound swabs, oropharyngeal or nasopharyngeal secretions.

5. Coagulase-negative Staphylococcus bacteria were excluded because of their association with sampling contamination unless strong clinical suggested and repeated positive cultures from the same patient implicated them.

\section{Methods}

All samples were collected, processed and identified under complete aseptic condition, using standard microbiological methods. For blood culture, 3 to $5 \mathrm{ml}$ of blood sample was obtained from a peripheral vein under aseptic precautions. Blood samples were incubated in blood culture incubator (BactAlert, BioMerieux, France).

Sputum, urine, body fluid, pus specimens were transported to the lab within 2 hours and were subjected to the following:

a- Inoculation on enriched media included: blood, MacConkey and chocolate agar plates. These were inoculated, incubated at $37{ }^{\circ} \mathrm{C}$, and examined for growth at 24-48 h. Isolates, if any, were identified by: Gram staining, colony characteristics, and biochemical properties. 
b- All samples were examined by Gram stain for microscopic examination.

A single colony of each morphotype was examined microscopically by Gram stain preparations, MALDI-TOF MS identification Samples Preparation (bioMérieux SA, France). Antibiotic sensitivity testing was done by (Vitek 2 system, BioMerieux SA, France).

Negative coagulase Staphylococcus bacteria were excluded because of their association with sampling contamination unless repeated positive cultures from the same patient implicated them. Therefore, 237cultured bacterial isolates from total samples were obtained.

\section{Statistical Analysis}

The collected data were checked, entered and analyzed by using SPSS statistical version 17 package. Data were expressed as mean \pm SD for quantitative variable, number and percentage for qualitative one.

\section{RESULTS:}

The antimicrobial profile susceptibility of 237 positive bacterial cultures was analyzed. The mean age of studied patients was 58.5 \pm 7.4 years. Males accounted for $59.1 \%$ (140) of subjects with isolates. The most common cause of liver cirrhosis was Hepatitis C Virus (HCV) infection (90.3\%). Hepatitis B virus (HBV) accounted for $4.6 \%$, autoimmune hepatitis (AIH) for $2.1 \%$ and unknown causes for $3 \%$.

The majority of positive bacterial cultures were urinary tract infection (60.4\%), followed by sepsis (18.1\%), chest infection (9.3), spontaneous bacterial peritonitis $(8.4 \%)$, and other infections (3.8\%). Most bacterial isolates were gram negative organisms (GNO) (70.9\%), mainly E coli (43.9\%), while, among cultures with gram positive organisms (GPO) (29.1\%), Staphylococci hemolytic was the most common $(10.1 \%)$.Table1
The commonest causative organism of UTI is E. coli 75/143 (52.5\%) and K. Pneumonia 27/143 (18.9\%). Sepsis in the studied decompensated cirrhosis patients caused mainly by negative coagulase staphylococci 19/43 (44.2\%) and E. coli $8 / 43(18.6 \%)$. SBP caused mostly by E. coli $9 / 20$ and kl. Pneumonia reported in $7 / 20$, while empyema in our patients caused mainly by $\mathrm{E}$ coli $3 / 6$. Chest infection occurred mostly by E coli $8 / 16$, while skin infection caused mainly by $\mathrm{Kl}$. Pneumonia organism 6/9. Table1

GPO in the studied patients show high resistance to all pencillins and carbepenam and high susceptibility to Vancomycin (70.8-100\%), Nitrofurantoin (25-100\%), linezolid (25-100\%), Tigycyclin (87.5-100\%), and Quinopristin (7.1$85.7 \%)$.

GPO was mainly resistant to $2^{\text {nd }}$ generation Cephalosporin. $75 \%$ of Staphylococcus Aureus were sensitive to levofloxacin. Negative coagulase Staphylococcus bacteria were high sensitive to Vancomycin, Nitrofurantoin, linezolid, Tigycycline and Quinopristin. Table2

\section{Cephalosporin and Quinolones susceptibility of GNO}

GNO showed high resistance to $3^{\text {rd }}$ and $4^{\text {th }}$ generations of cephalosporin, while had accepted sensitivity to $2^{\text {nd }}$ generation. GNO were recorded resistance to the most of the generations of Quinolones.

GNO were recorded susceptible to Amikacin $(50-87.5 \%)$, while Colistin susceptibility was $16.7 \%-51.1 \%$ of GNO. Table 3

Resistant bacteria isolated were observed as Extended-spectrum beta-lactamases in $32.2 \%$ of Ecoli and Klebsiella Sp. Staphylococcus aureus were Methicillin resistant in $100 \%$ of cases. vancomycin-resistant enterococci were recorded in $21.4 \%$ of enterococci fecalis infected cases, while Multi-Drug resistant strains were observed in $83.3 \%$ of acinetobacter infection, and $25 \%$ of Pseudomonas infection included one septic case had extensive drug resistance (XDR). Table 4 
Table (1): This table shows the types of cultured microbes in different biological samples.

\begin{tabular}{|c|c|c|c|c|c|c|c|c|}
\hline & & BLOOD & URINE & ASCITES & PLEUAL & SPUTUM & PUS & TOTAL \\
\hline \multirow{7}{*}{$\begin{array}{l}\text { GNO } \\
\text { N=168 } \\
(70.9 \%)\end{array}$} & E. COLI & 8 & 75 & 9 & 3 & 8 & 1 & $104(43.9 \%)$ \\
\hline & KL. PNEUMONIA & 2 & 27 & 7 & 2 & 1 & 6 & $45(18.99 \%)$ \\
\hline & ACINTEOBACTER & 0 & 4 & 1 & 1 & 0 & 0 & $6(2.5 \%)$ \\
\hline & PROTEUS & 0 & 1 & 0 & 0 & 1 & 0 & $2(0.84 \%)$ \\
\hline & PSEUDOMONOAS & 3 & 4 & 1 & 0 & 0 & 0 & $8(3.4 \%)$ \\
\hline & ENTEROBACTER & 0 & 1 & 0 & 0 & 0 & 0 & $1(0.42 \%)$ \\
\hline & BURKHOLDERIA. & 0 & 2 & 0 & 0 & 0 & 0 & $2(0.84 \%)$ \\
\hline \multirow{8}{*}{$\begin{array}{l}\text { GPO } \\
\mathrm{N}=69 \\
(29.1 \%)\end{array}$} & $\begin{array}{l}\text { ST. } \\
\text { HAEMOLYTICS }\end{array}$ & 10 & 9 & 0 & 0 & 5 & 0 & $24(10.1 \%)$ \\
\hline & ST. AEURUS & 2 & 3 & 2 & 0 & 0 & 1 & $8(3.4 \%)$ \\
\hline & ST. HOMINIS & 9 & 1 & 0 & 0 & 0 & 0 & $10(4.2 \%)$ \\
\hline & ST. WARNERI & 0 & 1 & 0 & 0 & 0 & 0 & $1(0.42 \%)$ \\
\hline & ST. EPIDERMIDIS & 6 & 0 & 0 & 0 & 0 & 1 & $7(2.95 \%)$ \\
\hline & ENTEROFECIUM & 0 & 3 & 0 & 0 & 1 & 0 & $4(1.7 \%)$ \\
\hline & E. FECALIS & 2 & 12 & 0 & 0 & 0 & 0 & $14(5.9 \%)$ \\
\hline & $\begin{array}{l}\text { BACILLUS } \\
\text { CEREUS } \\
\end{array}$ & 1 & 0 & 0 & 0 & 0 & 0 & $1(0.42 \%)$ \\
\hline & TOTAL & $43(18.1)$ & $143(60.4)$ & $20(8.4)$ & $6(2.5)$ & $16(6.8)$ & $9(3.8)$ & 237 \\
\hline
\end{tabular}

GNO (gram negative organisms), GPO (gram positive organisms).

Table (2): Antimicrobial susceptibility for Gram positive bacteria isolated from positive cultures.

\begin{tabular}{|l|c|c|c|c|c|c|c|c|}
\hline & $\begin{array}{c}\text { S. haemol } \\
\mathbf{n}(\mathbf{2 4})\end{array}$ & $\begin{array}{c}\text { S.hominis } \\
\mathbf{n}(\mathbf{1 0})\end{array}$ & $\begin{array}{c}\text { S.epid } \\
\mathbf{n}(\mathbf{7})\end{array}$ & $\begin{array}{c}\text { S.aureus } \\
\mathbf{N}(\mathbf{8}) \%\end{array}$ & $\begin{array}{c}\text { S.warenii } \\
\mathbf{N}(\mathbf{1}) \%\end{array}$ & $\begin{array}{c}\text { Bacillius } \\
\mathbf{N}(\mathbf{1}) \%\end{array}$ & $\begin{array}{l}\text { E. fecium } \\
\mathbf{( 4 )} \%\end{array}$ & $\begin{array}{c}\text { E.fecalis } \\
(\mathbf{1 4}) \%\end{array}$ \\
\hline AMP & - & - & - & - & - & - & - & $1(7.1)$ \\
\hline IMP & $1(4.1)$ & - & - & - & - & - & - & -- \\
\hline MEM & $1(4.1)$ & - & - & - & - & - & - & - \\
\hline CEFOX & - & - & - & - & - & $1(100)$ & - & - \\
\hline CN & $5(20.8)$ & $4(40)$ & $6(85.7)$ & $2(25)$ & - & - & - & - \\
\hline CIP & $1(4.1)$ & $1(10)$ & $1(14.3)$ & $4(50)$ & - & - & - & $2(14.2)$ \\
\hline LEV & $1(4.1)$ & $1(10)$ & $2(28.6)$ & $6(75)$ & - & $1(100)$ & - & - \\
\hline MOXIF & $8(33.3)$ & $3(30)$ & $5(71.4)$ & $5(62.5)$ & - & - & $1(25)$ & $1(7.1)$ \\
\hline VA & $17(70.8)$ & $9(90)$ & $7(100)$ & $8(100)$ & $1(100)$ & $1(100)$ & $4(100)$ & $11(78.6)$ \\
\hline RD & $5(20.1)$ & $3(30)$ & $4(57.1)$ & - & - & - & - & $2(14.2)$ \\
\hline DA & $11(45.8)$ & $4(40)$ & $2(28.6)$ & - & - & - & - & - \\
\hline SXT & $9(37.5)$ & $4(40)$ & $5(71.4)$ & $2(25)$ & - & - & - & - \\
\hline F & $17(70.8)$ & $10(100)$ & $7(100)$ & $6(75)$ & $1(100)$ & - & $1(25)$ & $11(78.6)$ \\
\hline TE & $5(20.8)$ & $5(50)$ & $3(42.9)$ & $4(50)$ & - & - & $1(25)$ & $1(7.1)$ \\
\hline LZD & $18(75)$ & $9(90)$ & $7(100)$ & $3(37.5)$ & $1(100)$ & - & $3(25)$ & $12(85.7)$ \\
\hline TGC & $20(83.3)$ & $10(100)$ & $6(85.7)$ & $7(87.5)$ & $1(100)$ & $1(100)$ & $4(100)$ & $13(92.9)$ \\
\hline QUINOP & $16(66.7)$ & $8(80)$ & $6(85.7)$ & $6(75.5)$ & - & - & $1(25)$ & $1(7.1)$ \\
\hline DALFOP & $4(16.7)$ & $1(10)$ & - & - & - & - & - & - \\
\hline
\end{tabular}

AMP (Ampcillin), IMP (Impenim), MEM (Meropenam), CEFOX (Cefoxitin), CN (Cefotatan) , CIP (Ciprofloxacin), LEV (Levofloxacin), MXF (Moxifloxacin), VA (Vancomycin), RD, DA (Clindamycin), SXT (Trimethoprim/Sulfamethoxazole), F (Nitrofurantoin), TE (Tetracycline), LZD (linezolid), TGC (Tigycycline), QUINOP (Quinopristin), DALFO (Dalfopristin) 
Table (3): Antimicrobial susceptibility for Gram negative bacteria isolated from positive cultures.

\begin{tabular}{|c|c|c|c|c|c|c|c|}
\hline & $\begin{array}{c}\text { E. coli } \\
(104) \%\end{array}$ & $\begin{array}{c}\text { Klebsiella } \\
\text { (45)\% }\end{array}$ & $\begin{array}{l}\text { Proteus } \\
\text { (2)\% }\end{array}$ & $\begin{array}{c}\text { Pseudomonas } \\
(8) \%\end{array}$ & $\begin{array}{c}\text { Acinetobacter } \\
(6) \%\end{array}$ & $\begin{array}{c}\text { Burkholderia } \\
\text { (2)\% }\end{array}$ & $\begin{array}{l}\text { Enterobacter } \\
\text { cloacae (1)\% }\end{array}$ \\
\hline TZP & $1(0.96)$ & $1(2.2)$ & - & - & - & - & - \\
\hline PRL & $11(10.58)$ & $3(6.7)$ & - & $4(66.7)$ & $1(16.67)$ & - & $1(100)$ \\
\hline ATM & $4(3.8)$ & - & - & - & $1(16.67)$ & - & - \\
\hline IMP & $68(65.4)$ & $16(35.6)$ & & $3(37.5)$ & $5(83.3)$ & $1(50)$ & $1(100)$ \\
\hline MEM & $71(68.3)$ & $19(42.2)$ & $2(100)$ & $3(37.5)$ & - & $1(50)$ & - \\
\hline ETP & $61(58.65)$ & $11(24.4)$ & $1(50)$ & - & - & - & - \\
\hline $\mathbf{K Z Z} 1^{\text {st }}$ & - & - & - & - & - & $1(50)$ & - \\
\hline $\mathbf{C N} 2^{\text {nd }}$ & $53(50.96)$ & $21(46.7)$ & - & $2(25)$ & $6(100)$ & - & $1(100)$ \\
\hline CTX 3 $^{\text {rd }}$ & $1(0.96)$ & - & - & - & - & $1(50)$ & - \\
\hline $\mathrm{CRO} \mathrm{3}^{\text {rd }}$ & $2(1.9)$ & - & - & $1(12.5)$ & - & - & - \\
\hline $\mathrm{CAZ} \mathrm{3^{ \text {rd } }}$ & $2(1.9)$ & - & - & $2(25)$ & - & - & $1(100)$ \\
\hline FEP $4^{\text {th }}$ & $4(3.8)$ & - & - & $4(50)$ & $1(16.67)$ & $1(50)$ & $1(100)$ \\
\hline CIP & $11(10.85$ & $2(4.4)$ & - & $4(50)$ & $2(33.33)$ & - & - \\
\hline LVX & $3(2.88)$ & - & - & - & - & - & - \\
\hline MOXI & $5(4.81)$ & - & - & - & - & - & - \\
\hline OF & $1(0.96)$ & - & - & - & - & - & - \\
\hline FOSF & $43(41.35)$ & $9(20)$ & - & - & - & - & - \\
\hline AK & $91(87.5)$ & $28(62.2)$ & $1(50)$ & $4(50)$ & $1(16.67)$ & $1(50)$ & - \\
\hline TOB & $22(21.15)$ & $7(15.6)$ & $1(50)$ & $2(25)$ & $5(83.3)$ & - & $1(100)$ \\
\hline SXT & $20(19.23)$ & $15(33.3)$ & $1(50)$ & $2(25)$ & $1(16.67)$ & $1(50)$ & - \\
\hline $\mathbf{F}$ & $47(45.19)$ & $4(8.9)$ & - & - & - & - & - \\
\hline COLISTIN & $20(19.23)$ & $23(51.1)$ & - & $2(25)$ & $1(16.67)$ & - & - \\
\hline TGC & $37(35.58)$ & $11(24.4)$ & - & - & $4(66.67)$ & - & - \\
\hline MINO & $9(8.65)$ & $8(17.8)$ & - & $1(12.5)$ & $2(33.33)$ & $1(50)$ & - \\
\hline
\end{tabular}

TZP (Pipercillin/Tazobactam), CLX (Cloxacillin), PRL(Piperacillin), (ATM(Aztreonam), IMP(Imipenem), MEM (Meropenem), ETP (Etrepenam), FEP(Cefepime), CN (Cefotetan), , KZ (Cefazolin), CTX (Cefotaxime), CRO (Ceftriaxone), CRO (Ceftriaxone), (CAZ (Ceftazidime), Cip (Ciprofloxacin), Lvx (Levofloxacin), Moxi , OF (Ofloxacin), fosf (fosfomycin), AK (Amikacin), (TOB(Tobramycin), SXT (Trimethoprim/Sulfamethoxazole), f (Nitrofurantoin), Colistin, TGC (Tigycycline), Mino(minocycline)

Table (4): Resistant bacteria isolated in clinical samples from cirrhotic patients according to the type of biological sample.

\begin{tabular}{|l|l|l|l|l|l|l|c|}
\hline \multicolumn{1}{|c|}{ Disease } & Sepsis & UTI & Chest Infect & Emyema & SBP & Skin infect & $\begin{array}{c}\text { Total } \\
\%\end{array}$ \\
\hline $\begin{array}{l}\text { ESBcteria } \\
\text { Klebsiella Sp. }\end{array}$ & $2 / 10$ & $31 / 102$ & $5 / 9$ & $3 / 5$ & $4 / 16$ & $3 / 7$ & $\begin{array}{c}\mathbf{4 8 / 1 4 9} \\
(\mathbf{3 2 . 2})\end{array}$ \\
\hline MRSA & $2 / 2$ & $3 / 3$ & $-/-$ & $-/-$ & $2 / 2$ & $1 / 1$ & $\begin{array}{c}\mathbf{8} / \mathbf{8} \\
(\mathbf{1 0 0})\end{array}$ \\
\hline VR E. fecalis & $2 / 2$ & $1 / 12$ & $-/-$ & $-/-$ & $-/-$ & $-/-$ & $\begin{array}{c}\mathbf{3} / 14 \\
(\mathbf{2 1 . 4})\end{array}$ \\
\hline MDR acinteobacter & $-/-$ & $3 / 4$ & $-/-$ & $1 / 1$ & $1 / 1$ & $-/-$ & $\begin{array}{c}\mathbf{5 / 6} \\
(\mathbf{8 3 . 3})\end{array}$ \\
\hline MDR Pseudomonas & $1 / 3 \mathrm{XDR}$ & $1 / 4$ & $-/-$ & $-/-$ & $0 / 1$ & $-/-$ & $\begin{array}{c}\mathbf{2 / 8} \\
(\mathbf{2 5})\end{array}$ \\
\hline
\end{tabular}

ESBL (Extended-spectrum beta-lactamases), MERSA (Methicillin-resistant staphylococcus aureus), VR E. (vancomycin-resistant enterococci), MDR (Multi-Drug resistant), XDR (EXtensive Drug Resistant) 


\section{DISCUSSION}

We evaluated the demographics, microbiological profile and antibiotic susceptibilities from records of liver cirrhosis patients with infection admitted in the ICU of a tertiary hospital in Egypt.

In this study, HCV infection was the main cause of liver cirrhosis, a finding reported Naga, et al., 2019 [10].

In our hospital, the sources of bacterial isolates were urinary tract infection $60.4 \%$, sepsis $18.1 \%$, chest infection 9.3\%, spontaneous bacterial peritonitis $8.4 \%$, and others $3.8 \%$ (table 1), These results mirror those of Jalan et al., 2014, who reported the following frequencies: urinary tract infection (23\% - 41\%), spontaneous bacterial peritonitis $(20 \%$ - 35\%), pneumonia $(8 \%-14 \%)$, spontaneous bacteremia $(8 \%-21 \%)$ and skin infections $(6 \%-13 \%)$.

The majority (70.9\%) of positive bacterial isolates were gram negative. E. coli was the most common GNO in these patients, while negative coagulase staphylococci followed by Enterococcus fecalis were the most frequent among GBO. These findings are in agreement with a recent study on similar patients in Europe [11].

In our study, the most common multi-resistant bacteria were $\mathrm{E}$ coli, followed by K. pneumonia and $\mathrm{E}$ fecalis sp. Other common isolates were acinteobacter and $\mathrm{E}$ faecium. similar findings were reported by Fernández et al., 2018 [11].

We recorded a raised frequency of Extendedspectrum beta-lactamases (ESBL) producing E. coli and Klebsiella. This was in agreement with Costabeber et al., 2016 [12]. All isolated S. aureus were methicillin resistant, but had high sensitivity to Vancomycin. According to Hubbard et al., 2020. Escherichia coli and Klebsiella pneumoniae, resistant to piperacillin/tazobactam (TZP) but susceptible to carbapenems and 3rd generation cephalosporins, has developed [16].

Shockingly high levels of $3^{\text {rd }}$ generation cephalosporin resistance were observed, despite their use as the empirical first choice antibiotic in cases of spontaneous bacterial peritonitis and other infections in liver cirrhosis patients [13, 14]. Most blood culture isolates $(97.7 \%)$ and $100 \%$ of isolates from the respiratory secretions and pleural fluids, were resistant to 3rd generations resistant. Virtually all urinary isolates $(99.3 \%)$ were resistant to $3^{\text {rd }}$ generation cephalosporins. In ascitic fluid samples, $95 \%$ of isolates were resistant to these antibiotics [12].

As per the European Association for the Study of the Liver guidelines, 2010 (EASL 2010) 3rd generation cephalosporins remain the empirical therapy for community acquired infections; but, meropenem or piperacillin/tazobactam, +/glycopeptide should be used in nosocomial infections.

The present study is in harmony with other study reporting the high frequency of the multiresistant bacteria in cirrhosis patients and especially with respect to 3rd generation cephalosporins. The study therefore endorses the use of the broad spectrum antimicrobials, such as Carbapenems, as the initial treatment in decompensated liver cirrhosis patients suspected or confirmed to have infection [15].

The choice of empirical antibiotic therapy should be established not only by the severity and the infection source, but also, on local anti-microbial susceptibility profiles. Bacterial resistance profiles in Egypt hospitals must be regularly reconsidered, and antibiotics stewardship programs established to improve prescribing habits if prognosis for decompensated cirrhotic patients is to be improved.

\section{CONCLUSION}

Urinary tract infection is the most common infection in patients with decompensated liver cirrhosis, with the commonest etiology; E. coli is highly resistant to first line antibiotics. Gram positive organisms remain sensitive to Vancomycin. The prevalence of multi-resistant bacteria in decompensated cirrhotic patients is high in our setting. Third generation cephalosporins are unsuitable to be prescribed as empirical therapy.

\section{Ethical consideration:}

The study was retrospective from medical records conducted according the protocol approved by Review Board of the Faculty of Medicine of Zagazig University.

\section{Acknowledgment:}

We thank our colleagues who support us to complete this study.

Funding: None. 
Conflicts of interest: None.

\section{Abbreviations:}

ICU: Intensive Care Unit

SBP: Spontaneous Bacterial peritonitis

GNO: Gram Negative organisms

E. coli: Escherichia. Coli

XDR: Extensive Drug Resistant

AMR: Antimicrobial resistance

ABR: Antibiotic resistance

MDR: Multidrug resistant

PDR: Pan drug resistant

TDR: Totally drug resistant

SD: Standard Deviation

HCV: Hepatitis C Virus

HBV: Hepatitis B Virus

GPO/GPB: Gram Positive Organisms/ Gram

Positive Bacteria

ESBL: Extended Spectrum Beta Lactamase

\section{HIGHLIGHTS}

- Urinary tract infection is the common infection in decompensated cirrhosis patients

- Multi-resistant bacteria prevalence in decompensated cirrhotic patients is high

- Empirical cephalosporin is unsuitable in decompensated cirrhotic infections.

\section{REFERENCES}

1. CDC. "Antibiotic Resistance Questions \& Answers". Get Smart: Know When Antibiotics Work. Centers for Disease Control and Prevention, USA. 30 June 2009.

2. CDC. "About Antimicrobial Resistance Antibiotic/Antimicrobial Resistance - CDC". www.cdc.gov. 19 September 2017. Archived from the original on 1 October 2017.

3. Golkar Z, Bagasra O, Pace DG. Bacteriophage therapy: a potential solution for the antibiotic resistance crisis. J Infect Dev Ctries 2014; $8(2): 129-36$.

4. Luyt CE, Brechot N, Trouillet JL, Chastre J. Antibiotic stewardship in the intensive care unit. Crit Care 2014; 18(5): 480.

5. CDC. Centers for Disease Control and Prevention, Office of Infectious Disease Antibiotic resistance threats in the United States, 2019. Available online: The full 2019 AR Threats Report, including methods and appendices, is available online at www.cdc.gov/DrugResistance/ Biggest-Threats.html.
6. Bunchorntavakul C, Chamroonkul N, Chavalitdhamrong D. Bacterial infectons in cirrhosis: A critical review and practical guidance. World J Hepatol 2016; 8(6): 307-321.

7. Jalan R, Fernandez J, Wiest R, Schnabl B, Moreau R, Angeli P, et al. Bacterial infections in cirrhosis: A position statement based on the EASL special conference 2013. J Hepatol 2014; 60:1310-1324.

8. Fernández J, Tandon P, Mensa J, Garcia-Tsao G. Antibiotic prophylaxis in cirrhosis: good and bad. Hepatology. http://dx.doi.org/10.1002/ hep 2015.28330.

9. Merli M, Lucidi C, Di Gregorio V, Falcone M, Giannelli V, Lattanzi B, et al. The spread of multi drug resistant infections is leading to an increase in the empirical antibiotic treatment failure in cirrhosis: a prospective survey. $P L O S$ One 2015;10 e0127448

10. Naga M, Esmat E, Ahmed H, Abdel Samie R, Hamdy A, Naga Y. "Clinical Characteristics of HCV in Egyptian Patients". EC Gastroenterology and Digestive System 2019; 6.2: 168-179.

11. Fernández J, Prado V, Trebicka J, Amoros A, Gustot $\mathrm{T}$, Wiest $\mathrm{R}$, et al. the European Foundation for the Study of Chronic Liver Failure (EF Clif), Multidrug-resistant bacterial infections in patients with decompensated cirrhosis and with acute-on-chronic liver failure in Europe, Journal of Hepatology 2018, doi: https://doi.org/10.1016/j.jhep.2018.10.027.

12. Costabeber AM, Mattos AA, Sukiennik TCT. Prevalence of bacterial resistance in hospitalized cirrhotic patients in Southern Brazil: a new challenge. Rev Inst Med Trop Sao Paulo 2016; $58: 36$.

13. European Association for the Study of the Liver. EASL Clinical Practice Guidelines for the management of patients with decompensated cirrhosis. J Hepatol 2018 Aug;69(2):406-460. doi: 10.1016/j.jhep.2018.03.024.

14. Runyon BA, AASLD Practice Guidelines Committee. Management of adult patients with ascites due to cirrhosis: an update. Hepatology 2009; 49:2087-107.

15. Patel V, Williams R. Antimicrobial resistance in chronic liver disease. Hepatol Int 2020; 14(1):24-34.

16. Hubbard A, Mason, J, Roberts, P. Parry, M, Corless, C, Aartsen, J, et al. Piperacillin/tazobactam resistance in a clinical isolate of Escherichia coli due to IS26-mediated amplification of blaTEM-1B. Nat Commun 2020; $11,4915$. 
17. Baron, E, Miller, J, Weinstein, M, Richter, S, Gilligan, P, Thomson, R, et al. A guide to utilization of the microbiology laboratory for diagnosis of infectious diseases: 2013 recommendations by the Infectious Diseases Society of America (IDSA) and the American Society for Microbiology (ASM)(a). Clinical infectious diseases: an official publication of the
Infectious Diseases Society of America, 57(4), e22-e121.

18. Sunil K. Taneja, Radha K. Dhiman, "Prevention and Management of Bacterial Infections in Cirrhosis", International Journal of Hepatology, vol. 2011, Article ID 784540, 7 pages, 2011. https://doi.org/10.4061/2011/784540. 